\title{
Computer based ex-ante evaluation of the planned railway line between Copenhagen and Ringsted by use of a Decision Support System named COSIMA-DSS
}

\author{
K. B. Salling \& A. Landex \\ Centre for Traffic and Transport, Technical University of Denmark, \\ Denmark
}

\begin{abstract}
This paper presents an appraisal study concerning the extension of the main railway line between Copenhagen and Ringsted by use of a software system named COSIMA-DSS. The modelling system is based upon a multi-methodological approach combining a cost-benefit analysis together with a multi-criteria analysis. One of the key impacts in cost-benefit analyses is the time benefit which depends on the timetabling. The paper describes some of the challenges related to the calculation of the time benefits and how the time benefits can be implemented in the decision support system. At the end of the paper, results from the calculations using COSIMA-DSS are presented and discussed on the basis of a newly developed Danish manual on transport appraisal.
\end{abstract}

Keywords: timetabling, queuing time, value of time, Decision Support System, cost-benefit analysis.

\section{Introduction}

Due to congestion it has been planned to increase the capacity of the main line between Copenhagen and Ringsted since the beginning of the 1990s. There are two different strategies to deal with the increase of traffic between Copenhagen and Ringsted, i.e. two more tracks along the existing railway line and a new railway line with a new layout cf. figure 1 . 
Due to increased traffic over the recent years, running times of the trains have been prolonged to obtain more capacity meaning longer travel times. The increase in time for the fast trains is significant, cf. figure i.e. on some travel relations the increase in time is up to $20 \%$ of the running time.

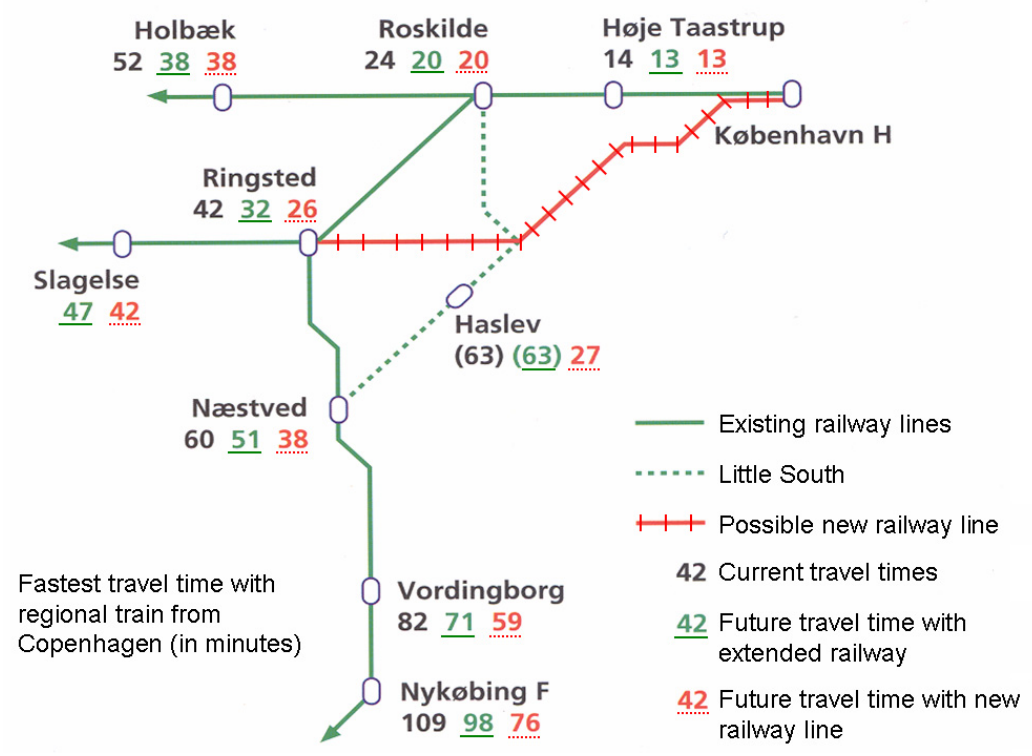

Figure 1: $\quad$ Fastest travel time with regional train from Copenhagen [11].

The decision whether to implement the new railway line or the extension is up to the decision-makers, in this case the Danish Government. However, by building a so-called decision support system (DSS), decision-makers can get "assistance" in making the best and most profitable choice from a societal point of view. It is necessary to stress that a DSS is not a correct and final answer to the problem it is merely assistance to the decision-makers.

\section{Time benefits}

In 2003 the Danish Ministry of Transport developed a set of guidelines on evaluating transport infrastructure projects [12]. When evaluating infrastructure projects using the Danish manual, the main impact group is the time benefits which in the evaluation of roads can make up a share in the range of $70-90 \%$ of the total benefits [7]. Therefore, it is important to make the correct assessment of the time benefits hence using the same guidelines in all transport projects for comparisons.

In schedule-based public transport systems, the time benefits can be divided into several elements - e.g. waiting time, transfer times, time spent in the vehicle and delayed time. Passengers consider each of these time elements differently 
and several studies have therefore been carried out to evaluate these [1]. For instance it feels worse to be delayed than to spend time in the vehicle - therefore, delayed time has a higher time value than ordinary travel time in the cost-benefit analysis (CBA).

The present timetable for the railway line between Copenhagen and Ringsted has been developed, adjusted and improved over time. In this sense it is difficult and time consuming to plan an optimal timetable for a new situation with an alternative infrastructure. It is, however, important to plan the best possible timetable to achieve as much time benefit for the passengers and freight as possible. In addition to the previously mentioned time aspects, two other key elements should be taken into account, namely the queuing time between trains and the timetabling. These impacts are highly relevant when evaluating public transport systems; however, they are not applied in the manual.

\subsection{Queuing time}

The queuing time is a time element in the appraisal study worth noticing. Queuing time on railway lines occurs when the traffic intensity is close to the maximum capacity level due to e.g. mixed operation (slow and fast trains). When close to the capacity level, the operation speeds of the fast trains must/will adapt to the slower trains cf. figure 2. This will increase the travel time for trains that under free conditions could run at higher speeds.

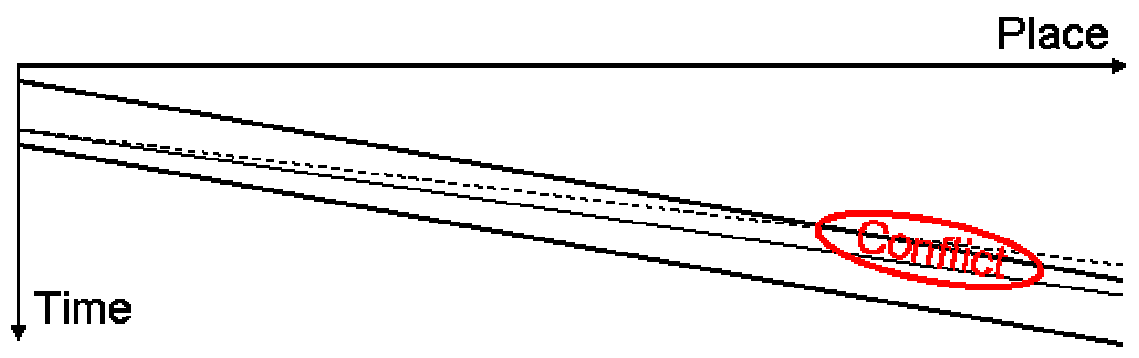

Figure 2: Extended running time (queuing time) due to other trains on the railway line.

The queuing time for the fast trains on the railway line between Copenhagen and Ringsted are significant, cf. figure 1. For some travel relations the queuing time is up to $20 \%$ of the running time.

Traditionally the queuing time is calculated as ordinary travel time for the passengers. However, it can be argued that the travel time should be divided into minimum travel time (including relevant time supplements) and queuing time. Furthermore, the queuing time should be assessed as a delay since the train and thereby the passengers are delayed due to other trains, even though it is a "scheduled delay". Whether the queuing time is calculated as ordinary travel time or delayed time, it has a great impact on the result, since normal delayed time are weighted twice as much as the ordinary travel time (for commuters 59 
DKK vs. 118 DKK [12]). The queuing time is an important factor particularly when making the timetables as illustrated in figure 2 .

\subsection{Timetabling}

Calculating the time benefits only by use of the traditional CBA can result in the paradox that a well-planned timetable gives a worse societal impact than a sloppily planned timetable, when for example an extension of a railway line is proposed. This paradox is due to the lower socio-economic cost of travelling when the queuing time is considered as ordinary travel time (Scenario 1) instead of delayed time (Scenario 2) which will occur when the queuing time is not taken into account, cf. table 1. In the future scenario (Scenario 3) a time cost per passenger is evaluated to be $1,188 \mathrm{DKK}$. Comparing the future scenario with the two basis scenarios the sloppily planned timetable actually performs better than the well planned timetable from a societal point of view.

Table 1: Traditional calculation of socio-economic time costs.

\begin{tabular}{|l|c|c|c|}
\hline & Scenario 1 & Scenario 2 & Scenario 3 \\
\hline Minimum running time (min) & 32 & 32 & 32 \\
\hline Queuing time (min) & 10 & 0 & 0 \\
\hline Travel time (min) & 42 & 32 & 32 \\
\hline Delay (min) & 0 & 10 & 0 \\
\hline Time costs per passenger (DKK) & 2,478 & 3,068 & 1,888 \\
\hline Difference future vs. basis & $\mathbf{5 9 0}$ & $\mathbf{1 . 1 8 0}$ & - \\
\hline
\end{tabular}

Converting some of the mentioned aspects into larger scale is always a great challenge especially as the infrastructure or timetabling is changed and often not known. The next section introduces the time aspect as it is calculated in a large scale network.

\subsection{Time benefits in large scale networks}

The time benefits can relatively easily be calculated for the existing timetable on the existing infrastructure e.g. table 1. It is, however, much more complex to calculate the time benefits when the infrastructure and/or the timetable is changed. The increased complexity of calculating the time benefits is due to the uncertainty on how the infrastructure and/or the timetable will look in the future.

Often more than one scenario is worked out and thereby also different candidate timetables. Since it takes a long time to create a timetable, the timetable is often only worked out for an analysis area. However, it is necessary to take the adjacent lines into account too because of the limitations of these line sections the so-called network effects [3, 4, 6] If the network effects are not included, only the local time benefits are examined. When the network effects are examined benefits from the rest of the railway network are included in the analysis. 
If a candidate timetable is only worked out for the analysis area it is not possible to calculate the time benefits precisely. One of the most difficult time effects to calculate is the queuing time. Previous studies on the queuing time $[3,4]$ have shown that the influence of the network effects can be significant for the queuing time.

The importance of the analysis area, and thereby the influence of network effects, can be illustrated by calculating the queuing time for both the whole of East Denmark and the analysis area only. The results are then made up as average queuing time per train $\mathrm{km}$, as the 3 main alternatives do not include the same number of train departures. The results appear from table 2.

Table 2: Queuing time in the Copenhagen-Ringsted project [3, 4].

\begin{tabular}{|l|l|c|c|}
\hline \multirow{2}{*}{ Area } & Basis & Extended line & New line \\
\cline { 2 - 4 } & \multicolumn{2}{|c|}{ (Queuing time in minutes per train km) } \\
\hline Whole East Denmark & 0.042 & 0.048 & 0.057 \\
\hline Only the analysis area & 0.033 & 0.046 & 0.033 \\
\hline $\begin{array}{l}\text { Analysis area in } \\
\text { proportion to East DK }\end{array}$ & $\mathbf{7 9 \%}$ & $\mathbf{9 6 \%}$ & $\mathbf{5 8 \%}$ \\
\hline
\end{tabular}

In both the basis and in the new line scenarios it is seen that the queuing time drops considerably, if it is only calculated locally as opposed to a bigger part of the network. An isolated local examination will therefore underestimate the queuing time when the project is seen in connection with the rest of the railway network.

In the extended line scenario with 4 tracks between Copenhagen and Ringsted the bottlenecks are now found on lines further away from the analysis area. This also reflects the amount of queuing time which is more or less the same $-96 \%$ of the queuing time per train $\mathrm{km}$ was found in the analysis area. In the extended line scenario there is therefore balance between the traffic and the infrastructure.

The queuing time analysis shown in table 2 indicates that the capacity conditions are underestimated when effects are only analysed locally. Furthermore, the results of the queuing time analysis shown in table 2 shows that it is important to include not only the analysis area but also the adjacent railway lines in the analysis of timetable scenarios since the queuing time otherwise might be underestimated.

Evaluating the Copenhagen-Ringsted project is especially difficult due to the previously mentioned time aspects. Further studies are necessary, especially when it comes to more widely applied effects e.g. network effects, queuing time and timetabling. These impacts are not yet applied in the Danish manual hence they are defined as strategic impacts. It is proposed to apply a multimethodology approach [8] both combining the conventional cost-benefit analysis (CBA) approach with a more widely multi-criteria analysis (MCA) approach. The first step is to see the problem as a system and hereby model the various strategic elements within the system in a more qualified way - this leads to a systematic approach/assessment e.g. the multi-methodology approach. 
The following section brings an overview of the applied multi-methodology approach together with the core impacts applied in this paper. Furthermore, the strategic impacts will be discussed as they are considered relevant for the Copenhagen-Ringsted railway line. Finally, all the effects are applied and evaluated in a socio-economic analysis.

\section{The appraisal framework - COSIMA-DSS}

The COSIMA-DSS (Omposite Model of Assessment-Decision $\underline{\text { Support }}$ System) model consists of two different modules brought together in the main module developed in Microsoft Excel [9]. The system shown in figure 3 gives a brief overview of the module structure of the model.

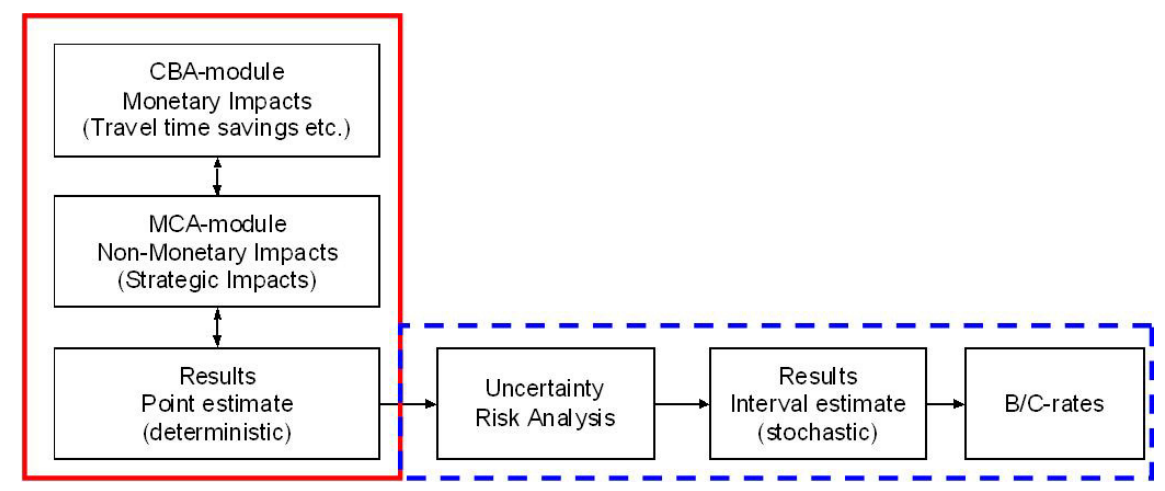

Figure 3: $\quad$ Main structure of the COSIMA-DSS model.

The left box consists of a so-called Value-Analysis module combining the traditional cost-benefit analysis (CBA) with the wider multi-criteria analysis (MCA). As shown in figure 3 the CBA module is of monetary character whilst the MCA module is of non-monetary character, in this case consisting of the previously mentioned queuing time and other strategic impacts as explained earlier. These different impacts (CBA and MCA) are combined in an Excel based model named COSIMA, determining a point estimate or rate of return, in this case by a benefit-cost ratio. The second box (on the right) is defined as the stochastic part that deals with the uncertainty present in all project appraisals. The uncertainty handling is performed by a Monte Carlo simulation which facilitates a complex analysis of the importance of uncertainty regarding some key input parameters. Furthermore, the project appraisal will have a more transparent perspective showing the degree of uncertainty for each element in the analysis ending up with an interval estimate as probability distributions [5]. In the following the deterministic box is applied to the Copenhagen-Ringsted framework. Recently, the stochastic modelling framework is not yet applied; however, in the future studies the stochastic calculations will be developed and implemented. 


\subsection{The CBA module}

The basic principle in the COSIMA framework is the cost-benefit analysis (CBA) which is based on the assumption that society's welfare is measured by the preferences of each individual and their presumed value [1]. CBA is traditionally used in Danish appraisal studies when it comes to infrastructure investments, however, in the public transport sector appraisal methodology is not widely applied. This is due to the complexities involved in the determination of the different impact groups and "actors". Traditionally, the main input in a socioeconomic analysis is the travel time savings, in evaluation schemes towards a railway line, this is only partly the case as several actors are involved, both operators and providers having to benefit from a new infrastructure investment. Furthermore, the strategic impacts such as the queuing time are important in order to make the overall performance of a railway network investment feasible as described in a previous section.

In the case of the Copenhagen-Ringsted railway line it is clear that by creating a new line or extending the existing line, the travel time will decrease meaning that the users will benefit from shorter travel times. Hopefully the operators will gain from an increase in travellers resulting in higher revenue and the providers will benefit from more travellers resulting in higher taxes etc. In figure 4 are the different impact groups together with their corresponding "actors" illustrated together with the benefit and cost groups.

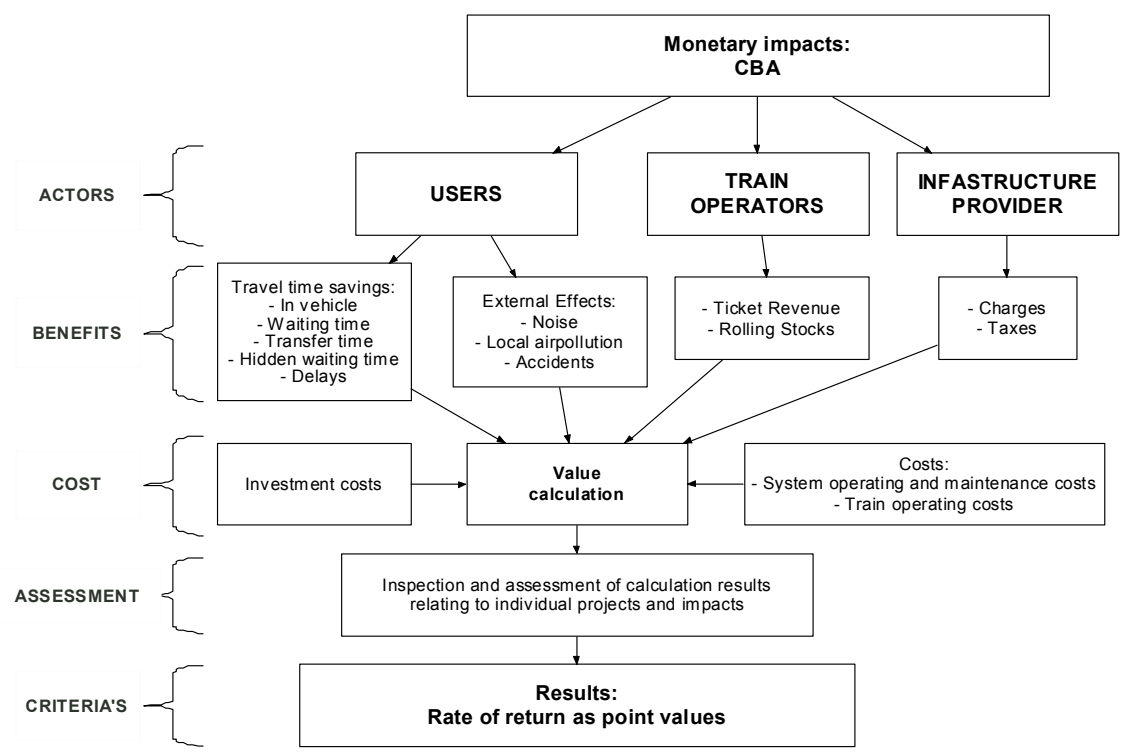

Figure 4: Flowchart on the different monetary impacts applied in the COSIMA-DSS model.

In the appraisal phase of the study, one general objective for the transport sector is to strengthen the competitiveness of the public transport as compared to 
the road sector. This should be done via different secondary objectives such as high service frequencies, low travel times, high service reliability, high comfort and good transfer possibilities to other transport modes (intermodality). These different groups are in some sense incorporated within the travel time savings, however, the CBA requires that all relevant impacts of the project are assigned a monetary value. In the case where different time impacts need to be taken into account these should be considered as well in the decision process. Various impacts considered of a strategic nature - e.g. long term environmental impacts or economic impacts can be difficult to quantify and thereby apply a unit price. In this sense a multi-criteria analysis (MCA) can help the decision-makers to apply these more strategic impacts in the evaluation scheme. When combining the CBA and MCA, a multi methodology assessment of the railway line is possible, in this case resulting in a benefit-cost ratio.

\subsection{The MCA module}

The multi-criteria analysis makes use of the well-documented AHP (Analytical Hierarchy Process) approach to pair-wise compare the various impacts $[2,10]$. Applying the pair-wise comparison makes it possible to assign a monetary unit to the MCA impacts even when quantitative ratings are unavailable. The different alternatives are assigned a score for each MCA impact and then the three MCA impact are compared in a software system named Criterium Decision Plus (CDP) by the AHP procedure [5], see figure 5.

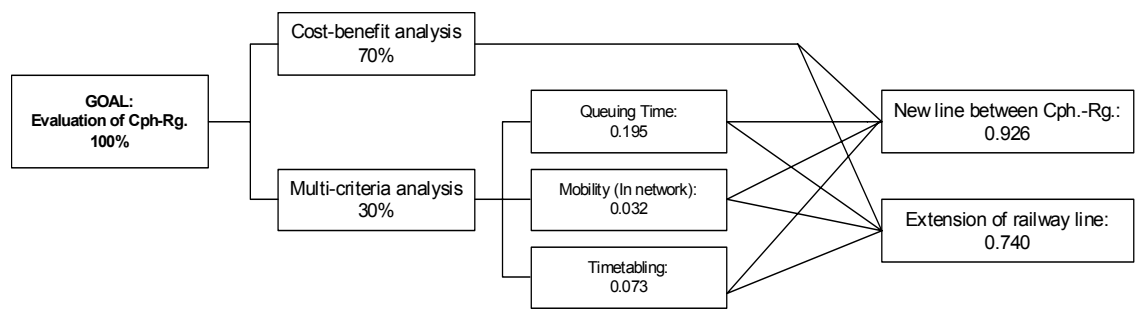

Figure 5: Model rates for the AHP procedure within CDP.

The output from the CDP is a set of normalized ratings of all the impacts which are transferred back to the CBA-module. The MCA impacts are then assigned a monetary value based on the comparison made in CDP. In this preliminary appraisal scheme it is assumed that the MCA counts for $30 \%$ and the CBA 70\% of all evaluated impacts. The strength of the pair-wise comparison is that it allows or incorporates the decision-makers in the overall decision process. In discussion with the decision-makers it has been decided that three non-monetary effects should be taken into account, namely the queuing time, the timetabling and the mobility concerning the large-scale network cf. figure 5 . The procedure is then to make the evaluation pair-wise considering all possible combinations between the three impacts. Finally, the two outer right boxes of the figure shows the preliminary ratings of the railway line - in this case it is the new line alternative which is merely better than the extension alternative. 


\subsection{Deterministic results}

By use of the methodology developed by the Danish Ministry of Transport [12], the $\mathrm{B} / \mathrm{C}$-ratios for the conventional CBA have been calculated as the bottom column in figure 6 . It is clear that the ratio for both alternatives is below 1.0 which means that none of the proposed alternatives are socio-economically feasible. However, as a result of the previous weighting schemes new calculations are performed to achieve total rate of returns (TRR) comprising both CBA and MCA.

\section{Total rate of return}

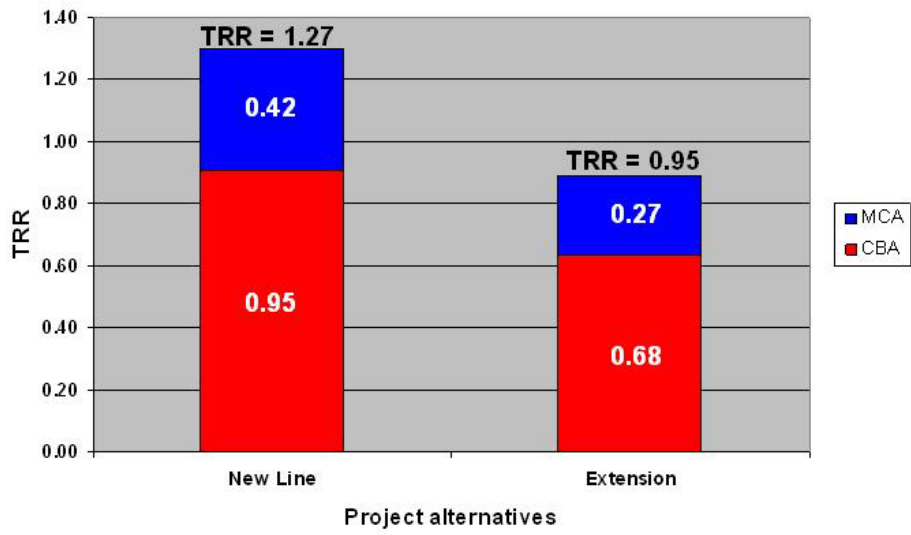

Figure 6: Graphic representation of a COSIMA-DSS calculation.

The COSIMA-DSS evaluation of the Copenhagen-Ringsted railway line shows that by assessing only two different alternatives the new line situation gives the best overall performance. Further development of the modelling scheme will be implemented as more alternatives are suggested.

Traditionally, evaluation methodology consists only of a cost-benefit analysis hence the bottom part of the columns shown in figure 5. However, none of the two ratios receives a positive rate of return which further concludes that the justification of implementing multi-criteria analysis is in order.

\section{Conclusions}

This paper has presented some of the challenges related to the calculation of time benefits on railway lines for socio economic analysis. It has been shown that the scheduled travel time for the passengers can be divided into minimum running time (including relevant supplements) and queuing time.

Furthermore, the paper has shown that the queuing time is difficult to calculate for future scenarios due to unknown timetables and network effects on large scale networks.

To deal with the difficulties of calculating time benefits the paper has presented a newly developed decision support system, COSIMA-DSS which 
aims at assisting decision-makers in the appraisal of transport infrastructure project investments. The variety of different features embedded within a CBA and MCA approach makes it particularly useful for addressing complex transportation decision problems. COSIMA-DSS gives the decision-makers a set of tools relevant for planning and assessment of project proposals where a conventional CBA will be too narrow a methodological approach. Further model work and more comprehensive case studies will seek to demonstrate and validate the COSIMA-DSS approach.

\section{References}

[1] Gissel, S., Decision aid methods in rail infrastructure planning, $P h D$. thesis at Department of Planning, Technical University of Denmark, 1999.

[2] Goodwin, P. \& Wright, G., Decision Analysis for Management Judgment. $2^{\text {nd }}$ edition, pp. 387-399, John Wiley \& Sons Ltd., Chichester, England, 1998.

[3] Hansen, S., Landex, A. \& A.H. Kaas, The Network effects of Railway Investments, Proc. of the $10^{\text {th }}$ International conference on Computers in Railways, eds. J. Allan, C.A. Brebbia, A.F. Rumsey, G. Sciutto, S. Sone \& C.J. Goodman, 2006.

[4] Hansen, S., Large Transport Infrastructure Investments and their Strategic Impacts with a Special Focus on Enterprises, PhD. thesis at Centre for Traffic and Transport, Technical University of Denmark, 2004 (in Danish).

[5] Infoharvest, Inc., Criterium Decision Plus - User's guide. Version 3, Infoharvest Inc., 1999, Seattle USA.

[6] Landex, A., Kaas, A.H., Schittenhelm, B. \& Schneider-Tilli, J., Practical use of the UIC 406 capacity leaflet by including timetable tools in the investigations, Proc. of the $10^{\text {th }}$ International conference on Computers in Railways, eds. J. Allan, C.A. Brebbia, A.F. Rumsey, G. Sciutto, S. Sone \& C.J. Goodman, 2006.

[7] Leleur, S., Road Infrastructure Planning - A Decision-Oriented Approach, 2nd Edition, Polyteknisk Forlag, Lyngby, Denmark, 2000.

[8] Leleur, S., Systemic Planning - Principles and Methodology for Planning in a Complex World, Polyteknisk Forlag, Lyngby, Denmark, 2004.

[9] Leleur, S., Holvad, T., Salling, K. B. and Jensen, A. V., Development of the CLG-DSS Evaluation Model. Midterm Report No. 1 - April 2004, Centre for Traffic and Transport - The Technical University of Denmark, 2004.

[10] Lootsma, F. A., Multi-criteria decision analysis via ratio and difference judgement, Kluwer Academic Publishers, 1999.

[11] The Danish Ministry of Transport, Upgrading the main railway lines Baneplansudvalget, April 1997 (in Danish).

[12] The Danish Ministry of Transport, Manual concerning socio-economic analysis - applied methodology in the transport sector, December 2004 (in Danish). 